Service social

\title{
Mouvance de la famille : réponses de l'action sociale, par Didier Le Gall et Claude Martin, Centre de recherche sur le travail social, Université de Caen, France, 1983, 340 pages.
}

\section{René Auclair}

Volume 34, numéro 1, 1985

Personnes âgées, milieux de vie et pratiques sociales

URI : https://id.erudit.org/iderudit/706266ar

DOI : https://doi.org/10.7202/706266ar

Aller au sommaire du numéro

Éditeur(s)

École de service social de l'Université Laval

ISSN

1708-1734 (numérique)

Découvrir la revue

Citer ce compte rendu

Auclair, R. (1985). Compte rendu de [Mouvance de la famille : réponses de l'action sociale, par Didier Le Gall et Claude Martin, Centre de recherche sur le travail social, Université de Caen, France, 1983, 340 pages.] Service social, 34(1), 196-197. https://doi.org/10.7202/706266ar d'utilisation que vous pouvez consulter en ligne.

https://apropos.erudit.org/fr/usagers/politique-dutilisation/ 
Nous l'avons déjà dit, pour les différents praticiens des services sociaux et de santé, ce volume arrive à point et permet de préciser les concepts et les approches de cette sphère de connaissance et de pratique. De plus, outre la partie décrivant des expériences, le volume a une orientation très concrète et on retrouve même, en annexe, des outils permettant l'analyse des réseaux et des quartiers.

La première partie du volume nous laisse toutefois plus songeur. Faut-il voir la bureaucratie et la récupération comme les seuls effets d'une plus grande prise en charge des services sociaux et de santé par l'État? A-t-on raison d'opposer le professionnalisme psychothérapeutique et l'aide naturelle, ou existe-t-il, à partir de ces pôles, un continuum de pratiques professionnelles et bénévoles qu'il faudrait identifier? Enfin, le retour de différentes formes d'aide naturelle est-il une manifestation d'une récupération en période de restrictions budgétaires ou l'indice de modifications sociales beaucoup plus larges?

École de service social,

Jocelyn LINDSAY

Université Laval.

Mouvance de la famille: réponses de l'action sociale, par Didier LE GALL et Claude MARtin, Centre de recherche sur le travail social, Université de Caen, France, 1983, 340 pages.

Cet ouvrage est le fruit d'une recherche-action auprès de femmes chefs de famille, effectuée à la demande d'une circonscription d'action sociale et associant professionnels du travail social et responsables administratifs de deux institutions : une Caisse d'allocations familiales et une Direction départementale de l'action sanitaire et sociale à Caen, en France. Son objet n'est pas seulement de faire état des conditions de vie ou de la quotidienneté des femmes chefs de famille, mais d'analyser leur situation en regard de l'action sociale afin de promouvoir un changement, tant des représentations que des pratiques.

C'est aussi, pour les auteurs, l'occasion d'une réflexion épistémologique sur ce mode d'intervention qu'est la recherche-action et sur sa capacité à produire du changement.

L'ouvrage est composé de trois parties. En premier lieu, on y précise l'objectif, la problématique et la méthodologie de la recherche. La description de la situation des femmes seules avec enfant(s) fait l'objet de la deuxième partie. Suivent enfin les propositions et les moyens d'action. La présentation de cette démarche de recherche-action est complétée par huit annexes et une bibliographie.

À notre avis, cette recherche appliquée est orientée vers la solution d'un problème urgent et important. Son but premier est d'apporter des solutions et des remèdes appropriés. Ses recommandations doivent permettre "de faire mieux, plus vite et à meilleur compte". 
La recherche-action est habituellement entreprise dans le but de transformer une situation ou dans celui, plus modeste, d'énoncer des recommandations qui, mises en œuvre, amèneraient un changement. Selon que son but immédiat est de susciter des changements (par des processus aussi divers que l'innovation, l'animation sociale ou le développement communautaire) ou d'étudier la situation pour éventuellement la transformer, les méthodologies peuvent varier considérablement (recherche, fonction mixte, animation sociale).

Rappelons qu'une intervention de changement peut habituellement être décrite à partir de quatre phases: diagnostic, planification, exécution et évaluation. Rappelons également que ces phases ne sont pas nécessairement des étapes, car les activités de l'une et l'autre peuvent souvent être concurrentes.

Nous avons trouvé, dans cet ouvrage, des réponses aux questions suivantes :

a) Identifier et décrire la situation insatisfaisante qu'on veut changer:

- Quelles sont les manifestations du problème?

- Quels sont les effets de cette situation?

- Qui vit cette insatisfaction?

- Par qui est-elle perçue comme étant insatisfaisante?

- Quel est l'historique de cette situation?

b) Décrire la situation qui serait désirée :

- Par qui est-elle désirée?

- Qui avantagerait-elle?

- Qui désavantagerait-elle?

Par contre, nous avons eu plus de difficulté à trouver des réponses aux questions relatives aux approches et aux stratégies de changement planifié.

a) Quelles sont les différents types d'approches à utiliser pour implanter le changement : empirico-rationnelle, normative-éducative, coercitive, consensuelle, conflictuelle ou contre-culturelle?

b) Par rapport à la situation qu'on veut changer, quelle forme pourrait prendre l'approche choisie?

c) En s'inspirant de l'ensemble de ces divers types d'approches, quels pourraient être les différents scénarios d'intervention?

En résumé, les auteurs de l'ouvrage ont mis plus l'accent sur les phases de diagnostic et d'exécution que sur celles de planification et d'évaluation dans leur compte rendu de recherche.

Ce livre s'adresse aux étudiants, aux travailleurs sociaux et à tous ceux qui, dans leur engagement social, cherchent une compréhension globale de leur action.

École de service social,

René AUCLAIR

Université Laval. 